\title{
Antibiotic resistance and plasmid carriage among Escherichia coli isolates from chicken meat in Malaysia
}

\begin{abstract}
Escherichia coli isolates from 131 raw chicken meat samples were tested for susceptibility to 12 antibiotics. Plasmids were isolated from many samples and their DNA molecular weight calculated. An $81.7 \%$ plasmid occurrence rate was observed among the isolates, ranging from 0 to 8 in number and with sizes from 1.2 to $118.6 \mathrm{MDa}$. Plasmids were detected in $93.8 \%$ of E. coli isolates resistant to all 12 antibiotics, and in $90.5 \%$ of E. coli isolates resistant to 11 . Three (2.8\%) isolates harboured 8 plasmids and were resistant to all 12 antibiotics. Antibiotic resistant genes in bacteria are usually carried in extrachromosomal DNA and it is postulated that E. coli with a high number of plasmids possesses wider resistance to antibiotics.
\end{abstract}

Keyword: Antibiotic resistant; Plasmid carriage; Escherichia coli; Chicken meat 